\title{
POLÍTICA INTERNACIONAL DE BENEDICTO XVI (19-4-2005/2013): LOS ACUERDOS CON LOS ESTADOS
}

\author{
Carlos Corral $^{1}$ \\ UNISCI / Universidad Complutense de Madrid (UCM)
}

\begin{abstract}
Resumen:
En el pontificado de Benedicto XVI se continuó la vía de acuerdos, convenios y concordatos con los Estados de todos los continentes, mediante las actualizaciones y ampliaciones consiguientes en virtud de los cambios operados en la realidad social, económica y política tanto de mundo en general como de Europa en especial. Se asiste a una consolidación de los acuerdos concordatarios y a su progresiva expansión a otros Estados.
\end{abstract}

Palabras clave: Benedicto XVI, concordato, acuerdos, expansión de los acuerdos.

Title in English: "Benedict XVI International Policy (19-4-2005/2013): Agreements with other States"

\begin{abstract}
:
Benedict XVI continued the signing of agreements and concordats with states from all continents, sometimes updating and upgrading them, given the changes in the social, economic and political domains that took place in the world and in particular in Europe. The article presents in detail the consolidated concordats and agreements that were signed during his pontificate and their gradual expansion to the different continents..
\end{abstract}

Keywords: Benedict XVI, Concordat, Agreements, Expansion of Agreements.

Copyright (C) UNISCI, 2013.

Las opiniones expresadas en estos artículos son propias de sus autores, y no reflejan necesariamente la opinión de UNISCI. The views expressed in these articles are those of the authors, and do not necessarily reflect the views of UNISCI.

\footnotetext{
${ }^{1}$ Carlos Corral Salvador es Catedrático Emérito de Fuerzas Religiosas en la Sociedad Internacional de la Universidad Complutense de Madrid, e Investigador Senior de UNISCI. Sus principales líneas de investigación son la libertad religiosa, el Derecho Eclesiástico del Estado, los fundamentalismos religiosos y los Concordatos de la Santa Sede. Dirección: C/ Universidad Comillas, 7, 28049 Madrid, España E-mail: ccorral@res.upcomillas.es.
} 


\section{Introducción}

Al tratar de ofrecer una retrospectiva de la política internacional de ocho años del pontificado de Benedicto XVI, resulta oportuno, creemos, dar a conocer cuál fue la política específica de acuerdos seguida con los Estados ${ }^{2}$.

Cierto que la saga de Países concordatarios existentes en 1939 antes del comienzo de la II Guerra Mundial — que al presente querían volver a serlo- quedó culminada con la "política" de acuerdos de Juan Pablo II, iniciada en 1978 y casi concluida años después en 1989 con la caída del muro de Berlín. De ahí que la política concordataria de Juan Pablo II apareciera configurada por dos características: la continuidad y la novedad.

Al presente, en cambio, con Benedicto XVI, la política de acuerdos con los Estados no puede ser otra que la continuidad matizada con alguna novedad. En el fondo, por tanto, se asiste a una consolidación de los acuerdos concordatarios y a su progresiva expansión a otros Estados.

\section{Consolidación y ampliación de los acuerdos concordatarios con los países germánicos}

En primer lugar, se produce una consolidación y ampliación de acuerdosos concordatarios con Baja Sajonia, Baviera, Hamburgo y Austria.

- Con Baviera ${ }^{3}$ se celebra su octavo convenio con la Santa Sede el 19 de enero de 2007, pero ahora bajo la figura de Protocolo Adicional al Concordato con Baviera de 29 de marzo de 1924, modificado por última vez por el Acuerdo de 8 de junio de 1988. Por él se introduce una nueva regulación del régimen de dotación de las Facultades de Teología en Baviera, debido a la disminución del número de estudiantes en los últimos años en el currículo de estudios para obtener el diploma y de los estudiantes de la disciplina de Religión Católica en algunas Facultades de Teología Católica y Centros de Instrucción de Baviera, que han conducido a una desproporción entre el número de docentes y el de estudiantes.

Como consecuencia, se han acordado una serie de medidas. La primera serie afecta a las Facultades de las Universidades de Bamberg y Pasau. Así se establece que estas quedan en estado de "suspensión" - por un período de quince años desde la entrada en vigor del Protocolo - tanto la obligación del Estado de corresponder a su enseñanza e instituir un currículo de los estudiantes de teología, como su obligación de proveer para la enseñanza del estudio en profundidad de la Religión Católica; que pueden reducirse a cinco el número de cátedras; y que así mismo, durante dicho período, se prescindirá de efectuar nuevos nombramientos.

La segunda serie afecta a las Facultades de las Universidades - cuatro- de Augsburg, Munich, (Ludwig-Maximilian-Universität), Ratisbona y Würzburg, por la que se mantiene la dotación numérica concordada de cátedras/plazas de profesor para filosofía y

\footnotetext{
${ }^{2}$ Corral, Carlos: "La política concordataria di Giovanni Paolo II”, Civiltà Cattolica (2001-IV), pp. 156-167.

${ }^{3}$ Corral, Carlos y Santos, José Luis (2012): Tratados internacionales (2003-2012) de la Santa Sede con los Estados, Concordatos vigentes, Madrid, Universidad Pontificia Comillas-, E-Book, pp.22-28.
} 
teología; y, en concreto, para Munich, 16 plazas; para Würzburg, 14; para Ratisbona, 12; y para Augsburg, 12.

- Con Baja Sajonia, mediante sucesivos acuerdos se había ido operando de modo conjuntado con la Iglesia Católica una continua adaptación del Concordato a las diversas reformas universitarias y escolares introducidas por el Land. Ahora se acomete sólo un punto: la adaptación relativa al status de las escuelas bajo titularidad de la Iglesia que tuvieren la categoría jurídica de Ersatzschule [las escuelas substitutivas] mediante el correspondiente Convenio de 6 de abril de $2010^{4}$.

- Con La Ciudad Libre y Hanseática de Hamburgo ha celebrado ya dos convenios, uno general y otro sectorial.

El primero fue el Convenio con la Santa Sede de 29 de noviembre de $005^{5}$. Su finalidad era "el deseo de Consolidar y desarrollar las relaciones entre la Iglesia católica y la Ciudad Libre y Hanseática de Hamburgo con espíritu de mutua cooperación en la libertad", y fijar y seguir desarrollando de manera estable las crecientes relaciones" (Preámbulo, párrafo 1). Como novedosa peculiaridad para con la Iglesia Católica, se añade todavía una segunda finalidad más general por desbordar el ámbito territorial de Hamburgo, "la aspiración a favorecer así también la construcción pacífica de una Europa siempre más estrechamente creciente" (Preámbulo, párrafo sexto).

Como presupuesto social se parte de que una "sociedad pluralista y una Ciudad cosmopolita que se concibe mediadora entre los pueblos" [Preámbulo párrafo 2] . $^{6}$.

El segundo fue el Convenio para la erección de un centro de formación para la Teología Católica y la Pedagogía de la Religión en la Universidad de Hamburgo ${ }^{7}$ (de 18 de mayo de 2010). Con respecto al profesorado, para el llamamiento como profesor, aun cuando se haga por la misma Universidad, se requerirá que, por parte del Arzobispo de Hamburgo, no se haya formulado excepción alguna (art. 4,1).

- Con Schleswig-Holstein ${ }^{8}$--donde los católicos son 173.130 dentro de una población de 2.837.373 - se alcanza el Acuerdo de 12 de enero de 2009 con la finalidad de "de consolidar y desarrollar las relaciones entre la Iglesia Católica y el Land en el espíritu de mutua colaboración en libertad"

- Con Austria, buscándose una vez más la actualización de la dotación, se da el paso al "Sexto Acuerdo Adiciona9l" (de 5 de marzo de 2009) en el que se fija la cantidad de 17.295.000 Euros, a partir del año 2008.

\footnotetext{
${ }^{4}$ Ibid., pp. 19-21.

${ }^{5}$ Ibid., pp 62-77.

${ }^{6}$ En cuanto a la estructura formal, el Convenios viene denominado en el original en alemán como "Vertrag", mientras en el original con la Santa Sede viene traducido como "Accordo" (en lugar de "Convenio" o "Convenzione"; en AAS “Conventio").

${ }^{7}$ Ibid. pp 78-84.

${ }^{8}$ Ibid. pp 85-99.

${ }^{9}$ Ibid., pp 111-112.
} 


\section{La incorporación de nuevos acuerdos con los estados egresados de la antigua Yugoslavia}

En Yugoslavia que sufrió sucesivos desmembramientos no fue posible concluir acuerdos con todos los nuevos Estados surgidos a la independencia. Tan sólo se consiguió con Croacia el 19 de diciembre de 1996 mediante acuerdos específicos. Ahora ya sí se han acabado de celebrar convenios con Albania, Bosnia-Herzegovina, Eslovenia y Montenegro.

- Con la República de Albania, al primer Acuerdo entre la Santa Sede y Albania para regular sus mutuas relaciones (de 23 de maro de 2002) ${ }^{10}$, se ha alcanzado la celebración del segundo: el Acuerdo internacional con la Santa Sede (de 3 de diciembre de 2007) ${ }^{11}$, con la intención de "regular algunas cuestiones de naturaleza económica y tributaria".

- Con Bosnia-Herzegovina -país de abigarrada complejidad étnica y religiosa- se ha llegado al Acuerdo Básico y Protocolo Adicional con la Santa Sede (de 19 de abril y 29 de septiembre de 2006) ${ }^{12}$ con ánimo de proteger la situación jurídica de la minoría católica, asentada juntamente con población islámica y ortodoxa ${ }^{13}$. Se advierte, ante todo, una tendencia a equiparar en lo posible los efectos civiles de instituciones religiosas con los de las instituciones paralelas estatales. Los temas del Acuerdo no distan mucho de los temas habituales concordados con otros países del área europea, como tampoco el planteamiento y las soluciones arbitradas a los mismos.

- Con la República de Lituania Acuerdo de 14 de junio de 2012 sobre reconocimiento de calificaciones concernientes a la educación superior.

- Con Montenegro se llegó a firmar el Acuerdo de Base con la Santa Sede, el 24 de junio de 2011, para la colaboración mutua y el establecimiento del marco general de las relaciones recíprocas ${ }^{14}$. Relaciones que tuvo lugar a los cinco años del reconocimiento, por parte de la Santa Sede, de la "vuelta de Montenegro a la Comunidad Internacional" (19 de junio de 2006) y del establecimiento de relaciones diplomáticas formales con el Vaticano (el 16 de diciembre de 2006.

La celebración de la firma del Acuerdo en el Vaticano, tuvo la particularidad que ocupó el centro de la audiencia que Benedicto XVI concedió al presidente del Gobierno de Montenegro, Igor Lukšić, quien lo firmó tras el encuentro con el Papa.

\footnotetext{
10 Los originales con introducción y versión en Corral, Carlos y Petschen, Santiago (2004): Tratados internacionales de la Santa Sede (1996-2003), Madrid, Universidad Pontificia Comillas; Concordatos vigentes, T.IV (Madrid) pp. 29-39.

${ }^{11}$ Santos, José Luis (2008): IUSTEL, RGDCDEE 17 (2008), pp. 1-16.

12 "Basic Agreement between the Holy See and Bosnia and Herzegovina (19 abril 2006)" y "Additional Protocol in the Basic Agreement between the Holy See and Bosnia and Herzegovina (29 septiembre 2006)", Ratificación, 25 octubre 2007. (Fuente: Nunciatura Apostólica de Bosnia Herzegovina).

13 Bosnia Herzegovina. Población 4.070.000: grupos étnicos: musulmanes, serbios, croatas; grupos religiosos: islámicos $40 \%$; ortodoxos $30 \%$; católicos $15 \%$; otras minorías religiosas $10 \%$.

${ }^{14}$ Santos, José Luis: "Montenegro, de mayoría ortodoxa, firma acuerdo con la Santa Sede (junio 2011)", Blog Carlos Corral, n.247, en www.periodistadigital.
} 


\section{Expansión a los países bálticos: Lituania}

A los cuatro países bálticos — que habían vuelto a celebrar nuevos Acuerdos con la Santa Sede como Polonia en 1993, Estonia en 1999, Letonia en 2000 y Lituania también en $2000^{15}$ — se suma al presente Lituania que ha firmado el 8 de junio de 2012 en el palacio de gobierno de Vilnius un acuerdo sobre el reconocimiento recíproco de los títulos de enseñanza superior (Agreement on the Recognition of Qualification Concerning Higher Education).

\section{Consolidación de acuerdos con los estados latinos}

Con menor incidencia e importancia, Andorra, España e Italia han celebrado los correspondientes Acuerdos con la Santa Sede, al que como colofón añadimos el de la Unión Europea.

- Con el Principado de Andorra se firmaba solemnemente, el 17 de marzo de 2008, en el Palacio Vaticano el Acuerdo con la Santa Sede ${ }^{16}$, por el por el Card. Secretario de Estado, y por el Jefe de Gobierno andorrano, Albert Pintat. Es un Acuerdo de carácter general que regula de conjunto las materias que de alguna forma afectan a la Iglesia y al Estado.

- Con España se firmaba un Canje de Notas Diplomáticas de Nunciatura-Ministerio, de 22 de diciembre de 2006, sobre la asignación tributaria a la Iglesia en España ${ }^{17}$.

- Con Francia, a sus Acuerdos anteriores, se añade ahora, primero, un Protocolo adicional [Avenant] de 12 de julio de 2005 a los convenciones diplomáticas de 14 de mayo de 1828 así como a los Protocolos adicionales de 4 de mayo de 1974 y 21 de enero de 1999, relativos a la iglesia y al convento de la Trinità in Monte ${ }^{18}$. Más tarde, un segundo Acuerdo de 18 de diciembre de 2008, relativo al reconocimiento de los grados y diplomas en la enseñanza superior por Francia ${ }^{19}$.

- Con Italia se recurre a un Canje de Notas sobre Procedimientos penales de clérigos, de 26 julio $2006^{20}$.

- Afectando a Europa, la Unión Europea, representada por la Comunidad Europea y por la República Italiana, y el Estado de la Ciudad del Vaticano, representado por la Santa Sede, han firmado un segundo Convenio Monetario el 17 de diciembre de $2009^{21}$ [que abroga el primero firmado el 29 de diciembre de 2000].

\footnotetext{
${ }^{15}$ Los cuatro Estados en Corral y Petschen, op. cit.

${ }^{16}$ Corral y Santos, op. cit., pp. 101-109.

${ }^{17}$ Ibid. pp. 179-182.

${ }^{18}$ Nota del 12 noviembre y 4 diciembre 2001, en "Bulletin officiel du ministére des affaires étrangéres", n 84 (30 septiembre 2003), p. 3; AA.VV. (2005): Liberté religieuse et régimes des eulte,s en droit franÇais, Paris, Cerf, pp. 811-812; En el “Journal Officiel de la République FranÇaise”, nº 26 (31 enero 2004), p. 2265 aparece lo siguiente: «Avis relatif á la publication des notes verbales relatives au statut des (Euvres pontificales missionnaires. NOR : MAEX0407 $1.1 \mathrm{~V}$. Les notes verbales relatives au statut des Guvres pontificales missionnaires échangées entre la Nonciature apostolique en France et le ministére des affaires étrangéres en date des 12 novembre et 4 décembre 2001 ont été publiées au Bulletin officiel do ministre des affaires étrangéres $n^{\circ} 84$ du 30 septembre 2003».

${ }^{19}$ Corral y Santos, op. cit., pp. 195-199.

${ }^{20}$ Ibid. pp. .201-227.

${ }^{21}$ Ibid pp. 263-268.
} 


\section{Expansión de los acuerdos a Brasil}

- Brasil ${ }^{22}$ : al tratarse de una de las mayores naciones del mundo como Brasil, se comprende el porqué Benedicto XVI recibió tan solemnemente en audiencia al presidente de la República Federal de Brasil, Luiz Inácio Lula da Silva, junto a su esposa y otros miembros de su séquito, para proceder a la firma del Acuerdo sobre el Estatuto jurídico de la Iglesia Católica en Brasil, el jueves 13 de noviembre de 2008. En efecto, es un País con una población de cerca de 186 millones sobre una superficie de $8.511 .965 \mathrm{Km}$., donde se encuentra establecida la casi la mayoría de las religiones y de las organizaciones religiosas. Según el censo de 2000, un $74 \%$ se declara Católico.

Los principios informadores del Acuerdo son los especificados en su Preámbulo: $-1^{\text {o } ~ " e l ~}$ servicio a la sociedad y al bien integral de la persona humana"; - $2^{\circ}$ la autonomía, la independencia y la soberanía de Iglesia y Estado, cada uno en su propio orden, y la mutua cooperación para la construcción de una sociedad más justa, fraterna y pacífica; $-3^{\circ}$ fundamentación, por parte de Brasil, en el propio ordenamiento jurídico y, por parte de la Iglesia, en el Concilio Vaticano Canónico.; $4^{\circ}$ reafirmación del "principio internacionalmente reconocido de libertad religiosa"; $5^{\circ}$ garantía del "libre ejercicio de cultos religiosos por la Constitución brasileña"; $6^{\circ}$ fortalecimiento y promoción de las "mutuas relaciones ya existentes".

Por ello, el presente Acuerdo solemne con Brasil tiene, entre otros, estos méritos: primero, es un acuerdo de carácter general, que regula todas las materias ordinarias de los concordatos vigentes; segundo, parte y desarrolla equilibradamente dos principios: el de aconfesionalidad (o laicidad) y el de cooperación; tercero, partiendo de la pluriculturalidad y pluralismo religioso, mantiene en las escuelas públicas la enseñanza religiosa (católica o no) como materia facultativa en los horarios escolares normales.

\section{Expansión a los estados africanos}

- Con Guinea Ecuatorial (antigua provincia de España) se llegó a la firma de un "Acuerdo sobre las relaciones entre la Iglesia Católica y el Estado" (el 13 de octubre de 2012), en el que viene establecido el marco jurídico y, en particular, la personalidad jurídica de la Iglesia y de sus instituciones, así como la asistencia espiritual a los fieles católicos en los hospitales y en las cárceles.

- Con Mozambique se logró establecer el marco jurídico de relaciones entre la Iglesia y el Estado en su Acuerdo con la Santa Sede, de 7 de diciembre de 2011, partiendo de la mutua independencia y autonomía y de mutua colaboración en bien de la población, en sectores comunes: salud, formación, educación, actividad asistencial.

\footnotetext{
${ }^{22}$ Ibid. pp. 139-148.
} 


\section{Expansión de acuerdos a estados asiáticos}

- Con Azerbaiyán ${ }^{23}$ — con muy amplia mayoría islámica (90\%) — se firmaba el Convenio con la Santa Sede (el 29 de abril de 2011) para garantízar el culto católico y la colaboración en el bien común de los ciudadanos.

- Con China [Taiwan], se celebró el Acuerdo entre la Congregación para la Educación Católica de la Santa Sede y el Ministerio de Educación de la República de China [Taiwan], de 2 de diciembre de 201, para la colaboración en el campo de la Educación Superior y el reconocimiento de estudios, títulos, diplomas y grados.

- Con Filipinas ${ }^{24}$ - con una población de 77 millones con el $84 \%$ de católicos y que no tiene concluido ningún Acuerdo general, sino tan solo un "Intercambio de Notas, de 20 de septiembre de 1951/1952, relativo a la erección del Vicariato Castrense en las Fuerzas Armadas" 25 - se procedió al presente a la firma de un Acuerdo de colaboración que tuvo lugar el 17 de abril de 2007 en la sede del Ministerio de Asuntos Exteriores en Manila, relativo al Patrimonio Cultural de la Iglesia. Su finalidad es "la salvaguarda, la valoración, y el disfrute de los bienes culturales" (art.III).

Como síntesis final, se constata que bajo el pontificado de Benedicto XVI se continúa la vía de acuerdos, convenios y concordatos con los Estados de todos los continentes, mediante las actualizaciones y ampliaciones consiguientes en virtud de los cambios operados en la realidad social, económica y política tanto de mundo en general como de Europa en especial ${ }^{26}$.

$$
+++
$$

Sin embargo, dicha saga de acuerdos/convenios concordatarios se fue completando hasta el final del pontificado con ulteriores Acuerdos firmados en 2001 con Eslovenia (11de octubre, Acuerdo sobre cuestiones jurídicas), Gabón, Acuerdo sobre el estatuto de la Enseñanza católica (26 de julio) y Suecia (24 noviembre, Canje de notas sobre personalidad de la Iglesia Católica); en 2002 con Albania (23 de marzo, Acuerdo para regular sus mutuas relaciones), en 2003 con Brandeburgo (12 noviembre, Convención [general]), Bremen (21 de noviembre, Convención [general]), Eslovaquia (21 de agosto, Acuerdo sobre educación católica), Malta 28 de febrero, Protocolo Adicional para mejorar la instrucción y educación religiosa en las escuelas estatales); en 2004 con Paraguay (24 de diciembre, Convenio sobre Asistencia religiosa a las Fuerzas Armadas), Portugal (18 de mayo, Concordato) ${ }^{27}$.

En efecto, tanto la Europa Oriental como la Occidental habían recuperado enteramente su libertad e independencia, y se iba avanzando trabajosamente hacia una más estrecha Unión Europea, por más que ahora se sienta aquejada de una grave crisis directamente económica e indirectamente política.

\footnotetext{
${ }^{23}$ Ibid. pp 113-116.

${ }^{24}$ Ibid. pp. 183-185.

${ }^{25}$ Corral y Petschen, op. cit., pp. 483-493.

${ }^{26}$ Añadamos una curiosa particularidad en las formalidades de la firma y/o ratificación de los Acuerdos, a saber, que Benedicto XI, al inicio de su pontificado, ha vuelto a la praxis de la lectura de los discursos al realizarse el intercambio del texto escrito de los acuerdos.

${ }_{27}$ Ver textos originales, con introducciones y traducciones en Corral y Petschen, op. cit.; "Concordatos vigentes", op. cit. T.IV; y Corral y Santos, op. cit., pp.22-28.
} 